\title{
Conocimientos, actitudes y prácticas frente a la tuberculosis en trabajadores del sector salud en municipios prioritarios de la Costa Pacífica colombiana
}

Health Workers' Knowledge, Attitudes and Practices Towards Tuberculosis in Priority Municipalities of the Colombian Pacific Coast

Conhecimentos, atitudes e práticas perante a tuberculose em trabalhadores da saúde em municipalidades prioritárias do litoral pacífico sul da Colômbia

Rocío Carvajal-Barona Enf, MPH, ${ }^{1}$ María Teresa Varela-Arévalo Ps MsC, ${ }^{2}$ Paula Andrea Hoyos Ps MgF, ${ }^{2}$ Eliana Sofía Angulo-Valencia Soc MPH, ${ }^{3}$ Carolina Duarte-Alarcón Ps ${ }^{2}$

Recibido: 19 de julio de $2013 \bullet$ Aceptado: 10 de junio de 2014

Doi: dx.doi.org/10.12804/revsalud12.03.2014.04

Para citar este artículo: Carvajal-Barona R, Varela-Arévalo MT, Hoyos PA, Angulo-Valencia ES, Duarte C. Conocimientos, actitudes y prácticas frente a la tuberculosis en trabajadores del sector salud en municipios prioritarios de la costa pacífica Colombiana. Rev Cienc Salud. 2014;12(3): 339-52. doi: dx.doi.org/10.12804/revsalud12.03.2014.04

\section{Resumen}

Objetivo: Caracterizar los conocimientos, actitudes y prácticas frente a la tuberculosis (TB) en los trabajadores de la salud que participan en los programas de control en municipios prioritarios de la Costa Pacífica colombiana. Materiales y métodos: Se realizó un estudio descriptivo de corte transversal en 38 trabajadores de la salud, que corresponden a la mayor parte de la población del personal con funciones en los programas de TB de 10 municipios prioritarios en Nariño, Colombia. El instrumento utilizado fue un cuestionario estructurado, previamente validado a través de entrevistas cognitivas a personas con características similares a la población estudio, realizadas previamente a la recolección de información. Resultados: Los trabajadores reportan tener conocimientos sobre TB y su manejo de acuerdo a las normas de control establecidas. No obstante, se identificaron algunas prácticas de riesgo relacionadas con el cumplimiento de las medidas del plan de control de infecciones y actitudes que reflejan estigma frente a la enfermedad. Conclusiones: El estudio aporta información referente a los conocimientos, actitudes y prácticas del personal de salud en el pacífico nariñense, que se puede convertir en insumo de consulta al momento del

\footnotetext{
1 Departamento de Salud Pública y Epidemiología, Grupo de Investigación Salud y Calidad de Vida, Pontificia Universidad Javeriana Cali. 2 Departamento de Ciencias Sociales, Grupo de Investigación Salud y Calidad de Vida, Pontificia Universidad Javeriana Cali.

3 Profesora Programa de Sociología, Universidad del Pacífico. Correspondencia: mtvarela@javerianacali.edu.co
} 
diseño de estrategias de intervención que posibiliten el fortalecimiento del programa de control de TB con enfoque cultural en esta región.

Palabras clave: Tuberculosis, conocimientos, actitudes y prácticas en salud, trabajadores.

\section{Abstract}

Purpose: To describe knowledge, attitudes and practices of health workers of tuberculosis (TB) programs in priority municipalities of the Colombian Pacific coast. Material and Methods: A cross-sectional descriptive study was conducted with 38 health workers, corresponding to the entire population of people working at the TB programs in ten priority municipalities of Nariño, Colombia. A structured interview, previously validated trough cognitive interviews, was used. Results: Health workers report to have knowledge about TB and its management according to the established control guidelines. Nevertheless, some risk practices concerning biosecurity standards of infection control were found, that show some stigmatizing attitudes towards the illness. Conclusions: The study provides information about the health workers' knowledge, attitudes and practices towards TB in Nariño that can be used as input to the design of intervention strategies to strengthen the TB programs with local and cultural approach.

Key Words: Tuberculosis, Health Knowledge, Attitudes and Practices, Health Workers.

\section{Resumo}

Objectivo: caracterizar os conhecimentos, atitudes e práticas perante a tuberculose em trabalhadores da saúde que participam dos programas de controle em municipalidades prioritárias do litoral pacífico colombiano. Materiais e métodos: Realizou-se um estudo descritivo de corte transversal com 38 trabalhadores da Saúde, os quais correspondem a maior parte da população de pessoas com funções nos programas de TBC em 10 municipalidades prioritárias num departamento do sul da Colômbia. Usou-se um questionário estruturado, previamente validado através de entrevistas cognitivas realizadas em pessoas com caraterísticas similares as da população de estudo e realizadas previamente à coleta da informação. Resultados: Os trabalhadores disseram ter conhecimentos sobre a TBC e saber como tratar os pacientes com a doença seguindo as normatividades estabelecidas. Porém, identificaram-se algumas práticas de risco relacionadas com o cumprimento das medidas do plano de controle de infecções e atitudes que refletem o estigma perante a doença. Conclusão: $\mathrm{O}$ estudo confere informação a respeito dos conhecimentos, atitudes e práticas das equipes de saúde no pacífico sul da Colômbia, a qual se pode converter em insumo de consulta no momento do planejamento de estratégias de intervenção que possibilitem o fortalecimento do programa de controle de TB com enfoque cultural nesta região.

Palavras-chave: Tuberculose, conhecimentos, atitudes e práticas, trabalhadores. 


\section{Introducción}

La tuberculosis (TB) sigue constituyendo un problema social y de salud pública en el mundo. En el año 2009 se diagnosticaron 9,4 millones de casos y 1,7 millones de muertes por TB $(1,2)$. Aproximadamente, un tercio de la población mundial vive con el bacilo de la TB, si bien solo una parte de ellos desarrolla la enfermedad (3). En el año 2011, en Colombia, según datos del Ministerio de Salud (4), se registraron 11708 casos de tuberculosis, con una tasa de incidencia de 25 casos por cada 100000 habitantes (5).

Debido a su propagación, a los factores de riesgo asociados y a la carga de morbimortalidad asociada a la misma, se han generado múltiples estrategias para el control de la TB. A nivel mundial, una de las alternativas para ello ha sido la propuesta Stop to Tuberculosis desarrollada por la Organización Mundial de la Salud (OMS), en consonancia con los objetivos del milenio (6). Respecto a 1990 y para 2015, se pretende reducir en un $50 \%$ la prevalencia y mortalidad a causa de esta enfermedad, a través del acceso universal al diagnóstico y tratamiento, además de la protección de los grupos vulnerables a la TB.

En Colombia, se ha creado la estrategia $\mathrm{Co}_{\mathrm{O}}$ lombia libre de tuberculosis, la cual se ha implementado como alternativa de solución para su prevención, vigilancia y control, a través de la planeación de las actividades de control, de acuerdo a las funciones y responsabilidades de los actores del sistema de salud, adquisición, distribución y control de medicamentos y la asignación de recursos (económicos, técnicos y de talento humano) a los diferentes entes territoriales (5). Esta estrategia tiene como meta la curación de mínimo el 85 \% de los casos de TB, así como también la reducción de la incidencia, prevalencia y mortalidad asociadas. Lo anterior, se pretende alcanzar con el acceso oportuno al diagnóstico y tratamiento y con una mayor cobertura; así como también a través de la prevención de coinfecciones como el VIH.

A pesar de las estrategias implementadas y de los avances científicos sobre el tema, los conocimientos, las actitudes y prácticas frente a la TB siguen siendo un problema para su control, convirtiéndose en barreras para el diagnóstico y el tratamiento, no solo en la población afectada, sino también en el personal de salud que le atiende (7-10).

El desconocimiento de síntomas y formas de transmisión por parte de los trabajadores del sector salud con responsabilidades en la prevención y el control de la TB puede limitar la detección oportuna y facilitar la propagación de la enfermedad. Así mismo, la presencia de actitudes negativas en este personal, puede dificultar que los pacientes accedan a la atención, dado el estigma y la discriminación asociados a la enfermedad. A su vez, las prácticas de riesgo o de protección que tengan en el manejo de la TB (relacionadas con el uso de elementos de protección personal, la realización de exámenes médicos periódicos y el seguimiento de las normas y procedimientos en la atención a las personas con esta enfermedad) contribuirán o no al control de la misma (11-12).

Los estudios sobre los conocimientos que tienen los trabajadores de la salud sobre la TB muestran resultados variables. Algunos evidencian bajos niveles de conocimientos acerca de las causas de la enfermedad, los síntomas y el control de la infección, siendo los médicos quienes tienen mayores conocimientos en comparación con los enfermeros, el personal del laboratorio y de apoyo (13-19). Así mismo, se ha encontrado que los médicos del sector público, tienen mayores conocimientos que los que trabajan en el sector privado (18-20). Otros, por el contrario, han observado un nivel alto de conocimientos $(14,16,17,20-23)$. 
Los estudios sobre actitudes muestran en general actitudes negativas del personal hacia la persona con $\mathrm{TB}$, incluyendo el miedo a infectarse, relacionar la TB con el VIH/Sida, tendencia a aislar a los pacientes, la exclusión de algunas prácticas médicas, entre otras. Lo anterior puede generar una inadecuada atención a los pacientes y con esto obstaculizar el control y el tratamiento de la enfermedad $(7-9,11,13,14)$.

Así mismo, en cuanto a las prácticas que tienen los trabajadores de la salud en el manejo de la TB, se ha encontrado que tener un contacto frecuente con los pacientes y ser médico, enfermera y trabajar en las unidades médicas o en los laboratorios de microbiología, se relaciona con un mayor riesgo de adquirir la enfermedad $(11,12)$.

Aspectos como los conocimientos, las actitudes y las prácticas del personal de salud relacionado con la prevención y el control de la TB, podrían estar relacionados con los pobres resultados en las metas y programas en Colombia, debido a la importancia que tienen estos actores en la participación y el desarrollo de actividades fundamentales para hacer frente a la TB $(10,17$, $22,24-27)$. Estudios realizados en el país han encontrado una baja percepción de riesgo frente a la enfermedad, escasos conocimientos sobre la misma, alta aceptación social de los síntomas y signos de la enfermedad, estigmatización, poco apoyo social y bajos conocimientos en temas de diagnóstico, trasmisión y bioseguridad en el personal de salud, lo cual ha sido asociado en gran medida a la transmisibilidad de la enfermedad. No obstante, la mayoría de dichos estudios se han enfocado mayoritariamente en el tema de acceso y calidad de la atención en TB y son pocos los dirigidos a explorar los conocimientos, actitudes y prácticas del personal de salud al respecto (28-31).

De acuerdo con lo anterior, se desarrolló un estudio para caracterizar los conocimientos, actitudes y prácticas frente a la TB en el personal administrativo y asistencial del sector de la salud en 10 municipios prioritarios de la Costa Pacífica nariñense en Colombia. Esta región presenta el $15 \%$ de la carga total de TB del país y en ella se presentan problemas en la gestión del programa, reflejados en indicadores como abandono del tratamiento en el 10,5\% de quienes lo reciben, terminación del tratamiento en $70 \%$ de los casos y fracaso en el $2 \%$; indicadores que se encuentran por encima de los nacionales. Igualmente, en 2009, reportó el $36,3 \%$ de los casos de TB multidrogorresistente (MDR) del país (32).

Los resultados de este estudio permitirán generar propuestas para desarrollar estrategias de prevención y de control de la enfermedad que sean adoptadas e integradas al programa de control en los municipios de estudio, así como al Programa Nacional para el Desarrollo de Estrategias de Prevención y Control acorde a las realidades locales y culturales.

\section{Materiales y métodos}

El estudio fue descriptivo de corte transversal. Se evaluó a la mayor parte de la población de trabajadores de la salud involucrados con los programas de TB en instituciones de salud públicas y privadas de 10 municipios de la Costa Pacífica nariñense colombiana. Los participantes aceptaron participar en el estudio voluntariamente y diligenciaron el consentimiento informado. Como criterios de inclusión se consideraron tener funciones directas en los programas de TB y que no presentaran ninguna enfermedad mental conocida o discapacidad cognitiva que les impidiera responder el cuestionario. En total, participaron 38 trabajadores de la salud, y se presentó un rechazo a tomar parte del estudio por parte de 8 trabajadores.

La recolección de información se llevó a cabo entre marzo y abril de 2013 a través de 
un cuestionario construido para el estudio y fue sometido a entrevistas cognitivas para su ajuste. Por medio de estas se indagó a un grupo de profesionales si las preguntas eran claras y comprensibles, y fueron realizadas por una de las investigadoras principales con formación de Maestría en Salud Pública y experiencia con de grupos focales y entrevistas semiestructuradas. Para estas últimas se tuvieron en cuenta las intenciones de las preguntas, se realizó toda la entrevista y se identificaron preguntas problemáticas y, en ellas, la investigadora se detuvo e hizo una indagación más profunda. Las entrevistas cognitivas fueron grabadas y escuchadas por las entrevistadoras en el proceso de análisis y ajuste de cada pregunta. El cuestionario fue aplicado a través de entrevista por personal previamente entrenado y supervisado durante su realización.

Una vez se identificaron los trabajadores de la salud que cumplían con los criterios de inclusión, se contactaron vía telefónica para concertar una cita. En esta se les explicaron los objetivos del estudio, se obtuvo la firma del consentimiento informado y se realizó la aplicación del cuestionario.

En el estudio se tuvieron en cuenta las consideraciones éticas según la declaración de Helsinki y la Resolución 008430 de 1993 del Ministerio de Salud Nacional que regula la investigación en salud.

Las respuestas de los participantes fueron analizadas en el software Stata 11. Se realizaron análisis descriptivos de las variables y se cruzaron con la variable dedicación exclusiva al programa de TB.

\section{Resultados}

\section{Características de la muestra}

Las características de los participantes se resumen en la tabla 1. En total participaron 38 trabajadores de la salud de los 10 municipios incluidos, entre ellos auxiliares de enfermería (37\%), personal de laboratorio (26\%), enfermeras profesionales $(21 \%)$, promotores de salud (8\%) y médicos especialistas (8\%). El $81 \%$ fueron mujeres y el $19 \%$ hombres, con una edad promedio de 33,8 años ( $\mathrm{DE}=8,6)$, en un rango entre 20 y 57 años. Con respecto a su estado civil, el $53 \%$ eran solteros y el $42 \%$ casados o vivían en unión libre.

El tiempo promedio de experiencia en atención clínica fue de 8,2 años $(\mathrm{DE}=7,2)$ y el tiempo promedio de experiencia en la atención a pacientes con TB fue de 5 años ( $D E=5,2)$. El $53 \%$ tenían cargos asistenciales, el $31 \%$ eran coordinadores asistenciales y el $16 \%$ tenían cargos administrativos. El $52 \%$ tenían vinculación directa con la institución y el $42 \%$ por prestación de servicios. Solo el $34 \%$ de los funcionarios tenían dedicación exclusiva al programa de TB en la institución.

\section{Conocimientos, actitudes y prácticas}

frente a la TB

La mayoría de los trabajadores de la salud poseen los conocimientos esperados sobre TB, la forma de transmisión $(89,5 \%$ ) y causas de la misma $(97,4 \%)$, el tiempo en que una persona bajo tratamiento sigue transmitiendo la enfermedad $(94,7 \%)$, los grupos de personas en los que se debe realizar búsqueda activa de TB $(73,7 \%$ ), la gravedad de la enfermedad $(94,8 \%)$, la necesidad de realizar la prueba de VIH en la población infectada con TB $(89,5 \%$ ), cuándo solicitar un cultivo diagnóstico en TB (97\%), qué se considera como fracaso en el tratamiento $(78,9 \%)$, cómo hacer el diagnóstico de TB en niños menores de 5 años ( $82 \%$ ) y cuándo solicitar prueba de sensibilidad en TB (tabla 2).

En general, la mayoría de trabajadores con conocimientos inadecuados sobre la TB no tenían dedicación exclusiva al programa de TB; 
Tabla 1. Características de los trabajadores de la salud que participan en los programas de TB en instituciones de salud ( $\mathrm{n}=38$ )

\begin{tabular}{|c|c|c|}
\hline Característica & Frecuencia & Porcentaje \\
\hline \multicolumn{3}{|l|}{ Género $(n=36)^{*}$} \\
\hline Femenino & 29 & 80.56 \\
\hline Masculino & 7 & 19.44 \\
\hline \multicolumn{3}{|l|}{ Estado Civil $(\mathrm{n}=36)$} \\
\hline Soltero & 19 & 52.78 \\
\hline Casado & 11 & 30.56 \\
\hline Unión Libre & 4 & 11.11 \\
\hline Separado/Divorciado & 1 & 2.78 \\
\hline Viudo & 1 & 2.78 \\
\hline \multicolumn{3}{|l|}{ Formación } \\
\hline Auxiliar de enfermería & 14 & 36.84 \\
\hline Enfermera profesional & 8 & 21.05 \\
\hline Promotor & 3 & 7.89 \\
\hline Especialista & 3 & 7.89 \\
\hline Otro & 10 & 26.32 \\
\hline \multicolumn{3}{|l|}{ Cargo que ocupa en la Institución } \\
\hline Asistencial & 20 & 52.63 \\
\hline Coordinador Asistencial & 12 & 31.58 \\
\hline Administrativo & 6 & 15.79 \\
\hline \multicolumn{3}{|l|}{ Tipo de vinculación laboral } \\
\hline Directa & 20 & 52.63 \\
\hline Prestación de servicios & 16 & 42.11 \\
\hline Temporal & 2 & 5.26 \\
\hline \multicolumn{3}{|c|}{ Dedicación exclusiva al programa de TB } \\
\hline No & 25 & 65.79 \\
\hline Sí & 13 & 34.21 \\
\hline
\end{tabular}

* Se especifica el n en variables en las que hay datos perdidos.

el porcentaje de respuestas incorrectas a las preguntas realizadas fueron mayores en este grupo (tabla 2).

A continuación se describen los conocimientos y prácticas de los trabajadores respecto al manejo de la enfermedad, el seguimiento, los efectos adversos y las medidas para evitar el contagio de TB.

Con relación a los conocimientos sobre el manejo de la enfermedad, el $84 \%$ conocen el esquema de tratamiento indicado para casos nuevos de TB, pero solo la mitad ( $51 \%$ ) conocen el esquema para un caso definido como fracaso.

En cuanto a las actividades de seguimiento, la mayoría ( $97 \%$ ) creen que la visita domiciliar en los pacientes con diagnóstico de TB se debe realizar en todos los casos y la visita a los contactos al inicio del tratamiento, a los 6 y 12 meses de tratamiento, según el $84 \%$. Respecto a esto, el $70 \%$ hacen siempre visita domiciliar 


\section{Tabla 2. Conocimientos sobre la TB en trabajadores de la salud que participan en los programas de TB} en instituciones de salud ( $n=38$ )

\begin{tabular}{|c|c|c|c|c|}
\hline & & \multirow[t]{2}{*}{$\begin{array}{l}\text { Frecuencia } \\
\qquad(\%)\end{array}$} & \multicolumn{2}{|c|}{$\begin{array}{c}\text { Dedicación } \\
\text { exclusiva al } \\
\text { programa de TB } \\
(\%)\end{array}$} \\
\hline & & & $\mathrm{Si}$ & No \\
\hline \multirow{2}{*}{$\begin{array}{l}\text { La tuberculosis es } \\
\text { transmitida por }\end{array}$} & Bacteria * & $34(89.5)$ & 38 & 62 \\
\hline & Virus - hongo - parásito & $4(10.5)$ & 0 & 100 \\
\hline \multirow{5}{*}{$\begin{array}{l}\text { La Tuberculosis se } \\
\text { transmite por }\end{array}$} & Exposición a la tos de una persona infectada * & $37(97.4)$ & 32 & 68 \\
\hline & Exposición a risa, habla y canto de un enfermo & $12(31.6)$ & 50 & 50 \\
\hline & Contacto con sangre o fluidos corporales & $2(5.3)$ & 100 & 0 \\
\hline & Comer alimentos contaminados & $0(0)$ & 0 & 0 \\
\hline & Compartir implementos de cocina y aseo & $0(0)$ & 0 & 0 \\
\hline \multirow{4}{*}{$\begin{array}{l}\text { Una persona que está } \\
\text { en tratamiento para TB } \\
\text { sigue transmitiendo la } \\
\text { enfermedad cuando }\end{array}$} & Ha recibido menos de un mes de tratamiento ${ }^{*}$ & $36(94.7)$ & 36 & 64 \\
\hline & $\begin{array}{l}\text { La baciloscopia de control durante el tratamiento es } \\
\text { negativa }\end{array}$ & $2(5.2)$ & 0 & 100 \\
\hline & Ha recibido más de 4 semanas de tratamiento & $0(0)$ & 0 & 0 \\
\hline & Utiliza tapabocas & $0(0)$ & 0 & 0 \\
\hline \multirow{4}{*}{$\begin{array}{l}\text { Se debe realizar } \\
\text { búsqueda activa de } \\
\text { Tuberculosis en }\end{array}$} & Personas que han tenido contacto con pacientes con TB & $9(23.7)$ & 44 & 56 \\
\hline & Personas con diagnóstico de VIH & $10(26.3)$ & 40 & 60 \\
\hline & Personas con infecciones respiratorias & $4(10.5)$ & 50 & 50 \\
\hline & Todos los anteriores ${ }^{*}$ & $28(73.7)$ & 36 & 34 \\
\hline \multirow{3}{*}{$\begin{array}{l}\text { Gravedad de la } \\
\text { enfermedad }\end{array}$} & Muy grave & $24(63.2)$ & 37 & 63 \\
\hline & Grave & $12(31.6)$ & 25 & 75 \\
\hline & Poco grave & $2(5.2)$ & 50 & 50 \\
\hline \multirow{3}{*}{$\begin{array}{l}\text { Se debe realizar prueba } \\
\text { de VIH en personas } \\
\text { con TB }\end{array}$} & Siempre * & $34(89.4)$ & 38 & 62 \\
\hline & Casi siempre & $1(2.6)$ & 0 & 100 \\
\hline & Algunas veces / nunca & $5(7.9)$ & 0 & 100 \\
\hline \multirow{3}{*}{$\begin{array}{l}\text { Se considera un fracaso } \\
\text { en el tratamiento } \\
\text { cuando un paciente } \\
\text { tiene }\end{array}$} & Baciloscopia positiva al segundo mes de tratamiento & $10(26.3)$ & 30 & 70 \\
\hline & $\begin{array}{l}\text { A los seis meses de terminado el tratamiento vuelve } \\
\text { positivo a TB }\end{array}$ & $30(78.9)$ & 33 & 67 \\
\hline & Baciloscopia positiva al quinto mes o más de tratamiento * & $20(52.6)$ & 40 & 60 \\
\hline
\end{tabular}

* Conocimientos esperados.

a los contactos de los pacientes diagnosticados con TB. En relación a los motivos por los cuales no realizan dichas visitas, se mencionan principalmente los problemas de seguridad (58\%), la ausencia de esta actividad en su programación
(16\%) y la falta de acompañamiento (16\%). Igualmente, el $95 \%$ piensan que se debe realizar visita domiciliar en los pacientes abandonadores del tratamiento en todos los casos. Frente al abandono, el $73 \%$ afirman realizar 
visita domiciliar en los pacientes abandonadores. Las causas reportadas para no hacerlas son los problemas de seguridad (53\%), la falta de acompañamiento (16\%) y la ausencia de esta actividad en su programación (13\%).

El $82 \%$ de los trabajadores verifica constantemente la aparición de efectos adversos al medicamento en pacientes con TB. Entre estos, los que reconocen como los más frecuentes son el vómito y las náuseas (55\% en ambos casos), el dolor de estómago (42\%) y la ictericia (32\%). La conducta que toman ante los efectos adversos de los medicamentos principalmente es formular medicamentos para el manejo de síntomas (32\%). Un 8 \% manifiestan suspender el tratamiento por unos días frente a la presencia de estos efectos.

Por otro lado, con respecto al seguimiento de las medidas del plan de control de infecciones en el personal de salud para evitar el contagio de TB, el $100 \%$ afirman que cumplirlas es muy importante; considerando que las acciones que deben tomar al atender a una persona con TB son el lavado de manos después de manipular esputo $(97 \%)$, pasar por una evaluación médica cada dos años (87\%), el uso de mascarilla de alta eficiencia \# 95 para atender los casos de multidrogorresistencia (79\%), el uso del mandil largo y cerrado durante toda la atención (74 \%), realizar la entrevista a los pacientes en un ángulo de $45^{\circ}(66 \%)$ y en caso de tener cabello largo, mantenerlo sujeto hacia atrás (61\%).

El $81 \%$ afirma seguir las medidas del plan de control de infecciones al atender una persona con $\mathrm{TB}$, el $70 \%$ ha recibido capacitación acerca de estas normas y el $76 \%$ considera que su institución de salud las garantiza. No obstante, el $69 \%$ considera que es muy probable que se infecte con TB. Al comparar estos aspectos según la dedicación exclusiva o no al programa de TB, no se encontraron diferencias.
Por otro lado, en cuanto a la Guía de Atención de TB (33), el $84 \%$ afirma conocerla. Al respecto, el $76 \%$ afirma que mejora su desempeño como profesional, el $66 \%$ considera que brinda herramientas para todos los casos que atiende y el $58 \%$ que se ajusta a la experiencia práctica.

La mayoría afirma que la institución cuenta con la Guía (94\%), que esta es efectiva (79\%), importante (73\%), adecuada (71\%), eficiente (58\%) y necesaria (53\%). Así mismo, todos están de acuerdo en que está bien escrita y muchos consideran que mejora la atención (76 \%) y que es efectiva y precisa (63\%).

Con respecto al estigma frente a la enfermedad, el $54 \%$ afirma que tener TB no es una situación que genere vergüenza, para el $27 \%$ poca vergüenza, pero para el $11 \%$ mucha vergüenza. Para estos, tener TB sería muy grave en el $70 \%$ de los casos y poco o nada grave en el resto. El estigma tampoco se asoció con la dedicación exclusiva o no al programa de TB.

Por último, se evaluaron los conocimientos actitudes y prácticas frente a la educación del paciente con TB y su familia. El $66 \%$ afirma haber recibido capacitación al respecto; sin embargo, el $83 \%$ considera estar capacitado para hacerlo. Los temas que reconocen que deben ser abordados incluyen la sensibilización para el diagnóstico oportuno ante síntomas respiratorios, la importancia del tratamiento y su continuidad hasta el final y las medidas de protección para evitar el contagio (todos escogidos por un $89 \%$ ).

La mayoría (79\%) realiza educación a la población general que consulta a su institución de salud sobre TB cada tres meses y un $20 \%$ afirma no hacerlo nunca.

Con respecto a las actitudes de los trabajadores de la salud frente a la educación del paciente y su familia, se destaca que la mayoría está de acuerdo en que es interesante informar y educar, 
Tabla 3. Actitudes de los trabajadores de la salud hacia la educación del paciente con TB y su familia

\begin{tabular}{|c|c|c|c|c|}
\hline & $\begin{array}{l}\text { Totalmente de } \\
\text { acuerdo } \\
(\%)\end{array}$ & $\begin{array}{l}\text { De acuerdo } \\
\quad(\%)\end{array}$ & $\begin{array}{l}\text { En desacuerdo } \\
(\%)\end{array}$ & $\begin{array}{c}\text { Totalmente en } \\
\text { desacuerdo } \\
(\%)\end{array}$ \\
\hline $\begin{array}{l}\text { Es interesante informar y educar al } \\
\text { paciente y su familia acerca de la TB }\end{array}$ & 91,67 & 8,33 & 0 & 0 \\
\hline $\begin{array}{l}\text { Es incómodo atender a los pacientes de TB } \\
\text { con mascarilla }\end{array}$ & 36,11 & 27,78 & 16,67 & 19,44 \\
\hline $\begin{array}{l}\text { Es importante indagar sobre los efectos } \\
\text { adversos de los medicamentos en } \\
\text { pacientes con TB }\end{array}$ & 83,33 & 16,67 & 0 & 0 \\
\hline $\begin{array}{l}\text { En la atención a los pacientes de TB es } \\
\text { importante el uso de barreras protectoras } \\
\text { para evitar contagio }\end{array}$ & 61,11 & 25,00 & 11,11 & 2,78 \\
\hline $\begin{array}{l}\text { Son innecesarias las visitas domiciliares a } \\
\text { los pacientes diagnosticados con TB }\end{array}$ & 11,11 & 8,33 & 47,22 & 33,33 \\
\hline $\begin{array}{l}\text { En la entrevista es riesgoso realizar la } \\
\text { entrevista con el paciente de TB }\end{array}$ & 22,22 & 30,56 & 27,78 & 19,44 \\
\hline $\begin{array}{l}\text { Es satisfactorio educar al paciente sobre } \\
\text { su enfermedad }\end{array}$ & 83,33 & 16,67 & 0 & 0 \\
\hline $\begin{array}{l}\text { Es necesario realizar el examen de esputo } \\
\text { a los contactos del paciente }\end{array}$ & 77,78 & 16,67 & 2,78 & 2,78 \\
\hline $\begin{array}{l}\text { Es de mucho trabajo censar y visitar los } \\
\text { contactos de TB }\end{array}$ & 5,71 & 25,71 & 40,00 & 28,57 \\
\hline
\end{tabular}

es satisfactorio hacerlo y es importante indagar sobre los efectos adversos de los medicamentos. Consideran que en la atención a los pacientes de TB es importante el uso de barreras protectoras para evitar contagio, sin embargo, les parece incómodo atender a los pacientes con mascarilla. Para la mayoría es de mucho trabajo censar y visitar los contactos de TB (tabla 3).

\section{Discusión}

El presente estudio tuvo como propósito caracterizar los conocimientos, las actitudes y prácticas frente a la TB en el personal administrativo y asistencial del sector de la salud en 10 municipios de la Costa Pacífica nariñense en Colombia.

Si bien el estudio fue eminentemente descriptivo, sus resultados son valiosos en la medida en que se evaluó la mayor parte de los trabajadores de la salud involucrados en los programas de TB en dichos municipios, ubicados en una de las zonas de mayor incidencia, que aporta un importante porcentaje de casos de TB MDR del país y que presenta importantes limitaciones en la gestión del programa de control.

El estudio muestra adecuados conocimientos sobre la TB y su manejo en los trabajadores de la salud, lo cual coincide con los hallazgos en el tema de estudios internacionales $(14,16,17,20-23)$, pero contrasta con los estudios nacionales que señalan pobres conocimientos en esta población (29-31). Un alto nivel de conocimientos en los trabajadores de los municipios prioritarios se resalta como positivo, considerando que estos tienen un papel principal en el desarrollo de acciones para hacer frente a la TB y, por lo tanto, se podrían asociar con la detección oportuna, el manejo adecuado de los casos y la prevención de la transmisión de la enfermedad. 
No obstante, los conocimientos adecuados fueron mayores en aquellos trabajadores que tienen una dedicación exclusiva al programa de $\mathrm{TB}$, aspecto que ha comenzado a ser reportado por otros estudios similares $(25,31)$. Al respecto, se podría pensar que la multiplicidad de tareas a las que se enfrentan los trabajadores de la salud en Colombia y la exigencia de manejo de diferentes programas y servicios con distintos niveles de complejidad, limita que estos se especialicen en una sola enfermedad (o grupo de enfermedades similares) y desarrollen fortalezas para realizar adecuadamente su labor, afectando, por lo tanto, la calidad de su servicio.

Esta situación, sumada al hecho de que casi la mitad de trabajadores no tengan una vinculación directa con la institución o estén contratados temporalmente, podría estar afectando la motivación de los trabajadores, la ejecución del programa de TB y la continuidad de las actividades desarrolladas para tal fin. Resultados similares fueron reportados por un estudio sobre conocimientos, prácticas y actitudes en trabajadores de la salud en municipios del Valle del Cauca (31).

Frente a esto sería importante replantearse la necesidad de contratar personal dedicado a los programas de TB con condiciones laborales estables y que se les garanticen las prestaciones sociales y la afiliación a seguridad social; así como focalizar sus tareas, de manera que los trabajadores de la salud puedan recibir capacitación frecuente, tener mayor tiempo en su carga laboral para realizar acciones de educación y realizar actividades importantes que no logran llevar a cabo en los distintos programas que apoyan. A su vez, que se les garanticen los recursos necesarios para llevar a cabo su labor. Lo anterior encuentra sustento en los resultados del estudio, cuando los participantes señalan que no siempre pueden realizar visitas domiciliarias a los pacientes y a sus contactos, como tampoco a quienes abandonan el tratamiento o reinciden en la enfermedad, debido a las limitaciones de tiempo y a no lograr programar dichas actividades dentro de su planeación.

Por otro lado, si bien la mayoría de los trabajadores de la salud reportan seguir las medidas del plan de control de infecciones necesarias para la prevención de la transmisión de la TB, esto no se da en todos los casos; por eso resulta un importante riesgo que debe ser tenido en cuenta y hacia el cual se deben realizar acciones urgentes de intervención, teniendo en cuenta que la salud del trabajador se puede ver afectada y este, a su vez, podría transmitir la TB a otras personas (11-12).

Respecto a lo anterior, es importante señalar que no todos los trabajadores reportan haber recibido capacitación sobre las medidas del plan de control de infecciones y algunos manifiestan que sus instituciones de salud no las garantizan, lo cual coincide con los hallazgos de otros estudios en países de Latinoamérica $(26,27,31)$. Esto determina la necesidad de implementar con urgencia medidas que garanticen adecuados conocimientos y prácticas de protección en los trabajadores de la salud, a través del acceso a elementos de protección personal y condiciones de infraestructura adecuadas del puesto de trabajo.

Por su parte, la Guía de Atención de TB que deben seguir los trabajadores de la salud vinculados a los programas debe ser difundida regularmente por las instituciones de salud $\mathrm{y}$, adicionalmente, implementar mecanismos para su uso por parte de estos funcionarios de los programas.

De igual manera, considerando que algunos trabajadores afirman no conocer la guía, e incluso, no todos los que la conocen creen que brinde herramientas para atender los casos o que se ajuste a su experiencia práctica, se necesita documentar las particularidades de regiones que cuentan con una extensa zona rural y que presentan problemas en la implementación del Sistema de Salud por parte de la Coordinación 
Nacional del Programa Control de TB del Ministerio, de manera que este se pueda ejecutar mejor en dichas zonas. Resultados similares fueron reportados por el estudio en el Valle del Cauca, lo cual resulta preocupante en tanto pareciera constituir una percepción generalizada en el país, siendo esta guía de uso nacional (31).

Con respecto a las actitudes, un porcentaje importante de trabajadores afirma que la TB es una enfermedad que genera poca o mucha vergüenza, lo cual da cuenta de las actitudes negativas que tiene el personal de salud hacia la enfermedad. Los estudios señalan que el estigma en los trabajadores que atienden a esta población se asocia como una barrera que dificulta que los pacientes accedan a la atención o que sean atendidos adecuadamente, por lo cual, acciones educativas hacia los trabajadores de la salud serían importantes, en tanto les permitiría reflexionar respecto a sus creencias sobre la TB y el impacto de estas en su desempeño en el programa $(7-9,11,13,14)$.

Por último, si bien las actitudes frente a la educación del paciente y su familia parecen ser adecuadas, no todos los trabajadores de la salud han recibido capacitación para hacerlo, ni llevan a cabo acciones educativas y, para la mayoría, resulta difícil hacerlas en las casas de los pacientes y sus contactos, lo cual es indispensable para el control de la TB y la prevención de la transmisión. La sensibilización de los trabajadores de la salud en este aspecto resulta necesaria, de la mano con propuestas que hagan viable que este tipo de acciones puedan ser incluidas en sus planes de trabajo.

En conclusión, los resultados de este estudio señalan importantes focos de intervención que mejoren el desempeño de los trabajadores de la salud involucrados en los programas de TB en los municipios del litoral pacífico nariñense. Entre ellos se destaca la necesidad de mejorar las condiciones laborales y la dedicación al programa que afectan directamente el tipo, el tiempo y la calidad del servicio que prestan a los pacientes con TB y sus familias. Esto implica, a su vez, mayores garantías y capacitación para seguir las medidas del plan de control de infecciones. También se resalta como necesaria una revisión de la Guía de Atención de TB, de manera que esta se pueda ajustar a las necesidades de quienes la usan en los programas de TB.

El estudio aporta información valiosa sobre los conocimientos, actitudes y prácticas del personal de salud en el litoral pacífico nariñense, que permitirá contribuir en la construcción de estrategias de intervención que posibiliten el fortalecimiento del programa de control de TB con enfoque cultural y local.

No obstante, es importante tener en cuenta que el estudio presenta varias limitaciones metodológicas, relacionadas principalmente con el tamaño de la muestra que dificultó el análisis de los datos. Asimismo, el instrumento de recolección de información, por ser un cuestionario aplicado en forma de entrevista, puede estar sujeto al sesgo de reporte. Esto en la medida en que los trabajadores de la salud podrían haber reportado prácticas adecuadas y actitudes positivas dado el fenómeno de deseabilidad social.

\section{Agradecimientos}

Al Fondo Mundial de Lucha contra el Sida, la tuberculosis y la malaria, la Organización Internacional para las Migraciones de Colombia y la Pontificia Universidad Javeriana Cali por la financiación de esta investigación. Al Instituto Departamental de Salud de Nariño, a la Secretaría de Salud de Tumaco y los municipios en los cuales se llevó a cabo la recolección de información, a los trabajadores de salud de las comunidades de la Costa Pacífica nariñense por la participación en este estudio. A la Asociación Nacional de Alcaldes y Gobernadores de Municipios y Gobernaciones con Población Afrodescendiente (Amunafro) por ser socio estratégico y apoyo para el desarrollo local del proyecto de investigación. 
Descargos de responsabilidad Estudio financiado por el Fondo Mundial de Lucha contra el SIDA, la tuberculosis y la malaria, la Organización Internacional para las Migraciones de Colombia y la Pontificia Universidad Javeriana de Cali.

\section{Bibliografía}

1. Raviglione M. Global burden of TB and progress towards 2015 targets. Documento presentado en: $15^{\text {th }}$ Annual Conference IUATLD-NAR. 2011 feb 24-26; Vancouver.

2. Lönnroth K, Castro K, Chakaya MC, Chauhan LS, Floyd K, Glaziou P, et al. Tuberculosis control and elimination 2010-50: cure, care, and social development. Lancet. 2010;375:1814-29.

3. Organización Mundial de la Salud. 10 datos sobre la tuberculosis [internet]. 2014. [citado 2012 jun 1] Disponible en: http://www.who.int/features/factfiles/tuberculosis/es/index.html

4. Ministerio de Salud. ¿Qué es tuberculosis? [internet]. s. f. [citado 2012 jun 1] Disponible en: http:// www.minsalud.gov.co/salud/Paginas/Tuberculosis.aspx

5. Ministerio de la Protección Social, Instituto Nacional de Salud, Organización Panamericana de la Salud. Plan Estratégico Colombia Libre de Tuberculosis 2010-2015. Para la expansión y fortalecimiento de la estrategia Alto a la TB. [internet]. 2009. [citado 2012 jun 1] Disponible en: http://www.paho.org/col/ index.php?option=com_content\&view $=$ article $\& \mathrm{id}=431$ :plan-estrategico-colombia -libre- de-tuberculosis2010-2015\&catid=686\&Itemid $=361$

6. World Health Organization. The stop TB strategy. [internet]. 2006. [citado 2012 jun 1] Disponible en: http://whqlibdoc.who.int/hq/2006/WHO_HTM_STB_2006.368_eng.pdf

7. Moya EM. Tuberculosis and stigma: impacts on health-seeking behaviors and access in ciudad Juárez, México and El Paso, Texas. University of Texas at El Paso; 2010. 334 p.

8. Dodor EA, Neal K, Kelly S. An exploration of the causes of tuberculosis stigma in an urban district in Ghana. Int J Tuberc Lung Dis. 2008;12(9):1048-54.

9. Dodor EA, Kelly S, Neal K. Health professionals as stigmatisers of tuberculosis: insights from community members and patients with TB in an urban district in Ghana. Psychol Health Med. 2009;14(3):301-10.

10. Kiefer EM, Shao T, Carasquillo O, Nabeta P, Seas C. Knowledge and attitudes of tuberculosis management in San Juan de Lurigancho district of Lima, Perú. J Infect Dev Ctries. 2009;3(10):783-8.

11. Mathew A, David T, Thomas K, Kuruvilla PJ, Balaji V, Jesudason MV, et al. Risk factors for tuberculosis among health care workers in South India: a nested case-control study. J Clin Epidemiol. 2013;66(1):67-74.

12. He GX, Van denHof S, Van der Werf MJ, Wang GJ, Ma SW, Zhao DY, et al. Infection control and the burden of tuberculosis infection and disease in health care workers in china: a cross-sectional study. BMC Infect Dis. 2010;10(1):313-21.

13. Akin S, Gorak G, Unsar S, Mollaoglu M, Ozdilli K, Durna Z. Knowledge of and attitudes toward tuberculosis of Turkish nursing and midwifery students. Nurse Educ Today. 2011;31(8):774-9.

14. Thu A, Win H, Nyunt MT, Lwin T. Knowledge, attitudes and practice concerning tuberculosis in a growing industrialised area in Myanmar. Int J Tuberc Lung Dis. 2012;16(3):330-5.

15. Teixeira EG, Menzies D, Cunha AJ, Luiz RR, Ruffino-Netto A, Scartozzoni MS, et al. Knowledge and practices of medical students to prevent tuberculosis transmission in Rio de Janeiro, Brazil. Rev Panam Salud Publica. 2008;24(4):265-70.

16. Woith WM, Volchenkov G, Larson JL. Russian health care workers' knowledge of tuberculosis and infection control. Int J Tuberc Lung Dis. 2010;14(11):1489-92. 
17. Aguilar MJ, Cortés CS, Zenteno R. Conocimiento y actitudes sobre tuberculosis en personal médico de Veracruz, México. Med UNAB. 2008;11(3):213-7.

18. Al-Maniri AA, Al-Rawas OA, Al-Ajmi F, De Costa A, Eriksson B, Diwan VK. Tuberculosis suspicion and knowledge among private and public general practitioners: Questionnaire Based Study in Oman. BMC Public Health. 2008;8:177-184.

19. Vandan N, Ali M, Prasad R, Kuroiwa C. Assessment of doctors' knowledge regarding tuberculosis management in Lucknow, India: a public-private sector comparison. Public Health. 2009;123(7):484-9.

20. Dato MI, Imaz MS. Tuberculosis control and the private sector in a low incidence setting in Argentina. Rev Salud Publica. 2009;11(3):370-82.

21. Kanjee Z, Catterick K, Moll AP, Amico KR, Friedland GH. Tuberculosis infection control in rural South Africa: survey of knowledge, attitude and practice in hospital staff. J Hosp Infect. 2011;79(4):333-8.

22. Maciel ELN, De Araújo WK, Giacomin SS, De Jesus FA, Marques P, Dietze R. O conhecimento de enfermeiros e médicos que trabalham na Estratégia de Saúde da Família acerca da tuberculose no município de Vitória (ES): um estudo de corte transversal. Ciênc Saúde Coletiva. 2009;14(Supl. 1):1395-1402.

23. Yimer SA, Holm-Hansen C, Bjune G. Assessment of knowledge and practice of private practitioners regarding tuberculosis control in Ethiopia. J Infect Dev Ctries. 2012;6(1):13-9.

24. Hashim DS, Al Kubaisy W, Al Dulayme A. Knowledge, attitudes and practices survey among health care workers and tuberculosis patients in Iraq. East Mediterr Health J. 2003;9:718-31

25. Quintana B. Impacto de las reformas del sector de la salud sobre los recursos humanos y la gestión laboral. Rev Panam Salud Publica. 2000;8(1-2):43-54.

26. De Souza JN, Bertolozzi MR. The vulnerabilyte of nursing wokers to tuberculosis in a teaching hospital. Rev Lat Am Enfermagem. 2007;15(2):259-66.

27. Huaroto L, Espinoza MM. Recomendaciones para el control de la transmisión de la tuberculosis en los hospitales. Rev Peru Med Exp Salud Publica. 2009;26(3):364-9.

28. Cruz OA, Flórez EL, Muñoz AI. Conocimientos de la tuberculosis en trabajadores de la salud en una localidad de Bogotá DC. Av Enferm. 2011;XXIX(1):143-51.

29. Gaviria MB, Henao HM, Martínez T, Bernal E. Papel del personal de salud en el diagnóstico tardío de la tuberculosis pulmonar en adultos de Medellín, Colombia. Panam Salud Publica. 2010;27(2):83-92.

30. Laserson KF, Osorio L, Sheppard JD, Hernández H, Benitez AM, Brim S, et al. Clinical and programmatic mismanagement rather than community outbreak as the cause of chronic, drug-resistant tuberculosis in Buenaventura, Colombia, 1988. Int J Tuberc Lung Dis. 2000;4(7):673-83.

31. Carvajal R. Conocimientos, actitudes y prácticas relacionadas con la tuberculosis en profesionales de la salud del Valle del Cauca, Colombia 2010. Informe final de investigación. Cali: Secretaría de Salud Departamental del Valle del Cauca; 2011.

32. The Global Fund. Strengthen the STOP the TB strategy in 46 priority municipalities from Colombia [internet]. s. f [citado 2012 jun 1]. Disponible en: http://portfolio.theglobalfund.org/en/Grant/Index/ COL-011-G05-T

33. Colombia, Ministerio de Salud. Resolución 412, Por la cual se establecen las actividades, procedimientos e intervenciones de demanda inducida y obligatorio cumplimiento y se adoptan las normas técnicas y guías de atención para el desarrollo de las acciones de protección específica y detección temprana y la atención de enfermedades de interés en salud pública [internet]. (2000 feb 25) [citado 2012 jun 1]. Disponible en: http://www.saludcolombia.com/actual/htmlnormas/Res412_00.htm 